\title{
Retour d'expérience des attentats du 13 novembre 2015. Rôle d'une cellule de crise hospitalière
}

\section{Feedback on Terrorist Attacks on November 13, 2015. Role of a Hospital Crisis Unit}

\author{
A. Petit - S. Morel $\cdot$ E. Benmansour $\cdot$ M. Brossard Lahmy $\cdot$ M.-H. Lavollé $\cdot$ B. Terrine $\cdot$ A. Amselli $\cdot$ C. Sliwka $\cdot$ \\ A.-M. Veillerobe $\cdot$ D. Marcelin $\cdot$ P. Soubigou $\cdot$ J. Hubin $\cdot$ P. Tilleul
}

Reçu le 11 janvier 2016; accepté le 13 janvier 2016

(C) SFMU et Lavoisier SAS 2016

Résumé Les attentats du 13 novembre 2015 ont donné lieu à une mobilisation sans précédent des établissements hospitaliers. Le groupe hospitalier Pitié Salpêtrière, particulièrement impliqué dans la prise en charge des victimes, a activé durant cette nuit tragique son Plan Blanc et mis en application tous les principes préalablement définis permettant d'assurer un soutien logistique à la prise en charge médicale. La cellule de crise réunie autour du directeur de l'établissement a piloté cette mise en œuvre dans ses différentes dimensions : mobilisation des ressources humaines, activation de toutes les fonctions supports nécessaires (sécurisation du site, approvisionnements, ouverture de lieux d'accueil des familles et des personnels) et enfin gestion des familles. Rétrospectivement, cette mise en œuvre a été globalement conforme aux dispositions préétablies et efficace en termes de soutien à l'activité médicale d'urgence pratiquée. Elle

A. Petit $(\bowtie)$

Direction de la qualité,

de la gestion des risques et des relations avec les usagers,

hôpitaux universitaires Pitié Salpêtrière /Charles Foix,

Assistance Publique-Hôpitaux de Paris (APHP),

47-83 boulevard de l'Hôpital, F-75013 Paris

e-mail : agnes.petit@aphp.fr

S. Morel $\cdot$ E. Benmansour

Direction générale,

groupe hospitalier Pitié Salpêtrière/Charles Foix,

APHP, Paris

\section{Brossard Lahmy}

Direction des services économiques et logistiques,

groupe hospitalier Pitié Salpêtrière/Charles Foix, APHP, Paris

M.-H. Lavollé · B. Terrine

Direction des affaires médicales et de la stratégie,

groupe hospitalier Pitié Salpêtrière/Charles Foix, APHP, Paris

\section{A. Amselli}

Direction des ressources humaines,

groupe hospitalier Pitié Salpêtrière/Charles Foix, APHP, Paris suggère cependant une analyse critique des points de faiblesse afin d'améliorer les procédures du Plan Blanc, dans un contexte actuel que l'on sait particulièrement à risque.

Mots clés Plan Blanc $\cdot$ Cellule de crise $\cdot$ Afflux massif de victimes

\begin{abstract}
Terrorist attacks of November 13, 2015 resulted in a massive rallying in hospitals. The Pitié-Salpêtrière hospital group, particularly involved in the nursing of victims, activated its "Plan Blanc" during that tragic night and applied all previously set principles to provide logistical support to the medical care. The crisis unit gathered around the managing director of the establishment managed this implementation in various dimensions: human resources mobilization, activation of all necessary support functions (security of the site,
\end{abstract}

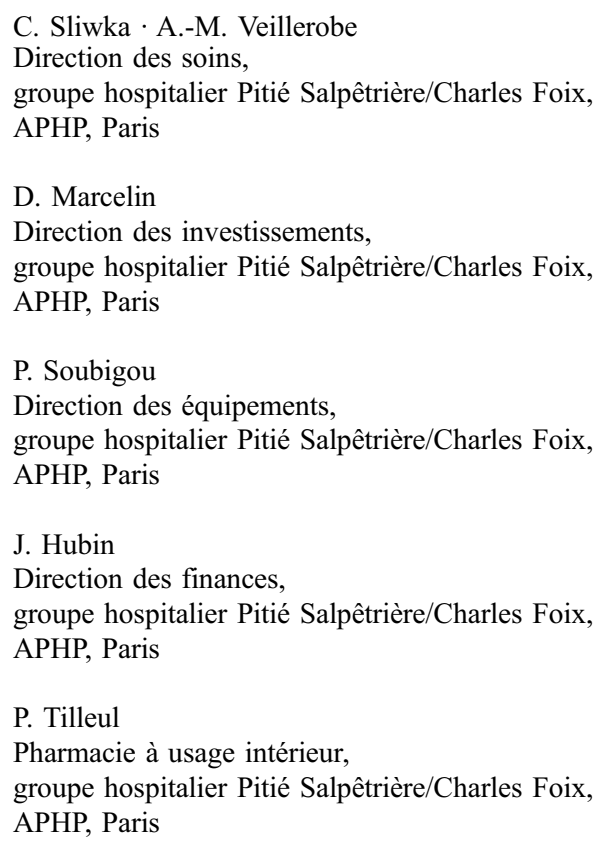


supply of provisions, opening of reception areas for families and staff) and finally, the management of families. Retrospectively, this implementation was globally in accordance with the pre-established dispositions and efficient in terms of support to the emergency medical activity.

However, it suggests a critical analysis of weak points in order to improve the procedures of the "Plan Blanc" in a particularly risky current context, as we know.

Keywords Plan Blanc $\cdot$ Hospital crisis unit · Mass casualties

\section{Introduction}

Le vendredi 13 novembre 2015, peu avant 22h, le groupe hospitalier Pitié Salpêtrière a activé sa cellule de crise, après l'annonce d'attaques terroristes multisites dans Paris et à Saint-Denis. À 22h34, l'Assistance Publique-Hôpitaux de Paris (APHP), sur décision de son Directeur Général, déclenchait le Plan Blanc.

Au cours de cette nuit, l'hôpital de la Pitié-Salpêtrière a reçu 53 victimes des attentats, dont 28 en urgence absolue. En l'espace de quelques heures, la mise en œuvre du plan NoVi-H (nombreuses victimes hospitalisées) appuyé par l'activation du Plan Blanc du site a permis une prise en charge des blessés d'une ampleur exceptionnelle [1] : plus du tiers des urgences absolues causées par les attentats de Paris a ainsi pu être accueilli, dix salles d'opération ont été armées et plusieurs centaines de personnels médicaux et paramédicaux ont répondu à l'appel. De l'avis des professionnels impliqués et des pouvoirs publics, c'est une médecine de pointe qui a répondu, dans un délai court, à des blessures de guerre. La qualité de la prise en charge des victimes a été largement soulignée, fruit d'une mobilisation et d'une organisation particulièrement efficaces. L'implication des équipes de direction de l'établissement dans la gestion de la crise a été également très forte.

À la tête de la cellule de crise, le directeur du groupe hospitalier et son équipe ont assuré la coordination des fonctions supports, nécessaires à la prise en charge des victimes. Il est ainsi proposé, au travers de cet article, d'analyser de manière critique les conditions dans lesquelles le Plan Blanc a été mis en œuvre afin d'y apporter tous les ajustements nécessaires.

\section{Organisation générale du Plan Blanc}

Le Plan Blanc est un élément fondamental de gestion des crises de grande ampleur [2-4]. Il décrit en effet une organisation destinée à répondre à tous les types de situations, quelles qu'en soient la nature (attentat, accident, événement cli- matique, épisode infectieux...), l'ampleur, l'évolutivité ou la durée. Du fait de la diversité des situations de crise possibles, le plan blanc est constitué d'un socle commun permettant la maîtrise des fonctions, dites "supports ", et de déclinaisons spécifiques en fonction du type de crise (plan NoVi-H pour l'accueil de nombreuses victimes, plan NRC pour les risques nucléaires, radiologiques ou chimiques, plan d'accueil des patients suspects ou atteints de virus hautement pathogènes [Ebola ou Mers-Corona Virus], plan canicule, plan inondation...).

Le socle commun du Plan Blanc décrit les principes (Fig. 1) permettant de :

- déclencher l'alerte et activer la cellule de crise ;

- sécuriser le site (fermeture et contrôle des accès, déploiement de vigiles) ;

- mobiliser les ressources humaines médicales et non médicales ;

- accueillir les patients (il s'agit dans ce cas de dispositions générales en appui des plans spécifiques [type plan NoVi-H]) ;

- assurer les fonctions logistiques d'approvisionnements des services cliniques et d'accueil et d'hébergement des personnels mobilisés ;

- accueillir et informer les familles des patients pris en charge, ou les familles à la recherche de leurs proches (ouverture d'un lieu dédié à l'accueil des familles);

- assurer la communication interne et externe ;

- gérer la fin de la crise.

La mise en œuvre des différentes fonctions est décrite ciaprès, ainsi que les axes d'amélioration identifiés.

\section{Mobilisation des ressources humaines}

\section{Organisation et mise en œuvre}

Le plan blanc repose sur une cellule de crise chargée de coordonner l'ensemble des fonctions nécessaires au traitement de l'évènement. A minima, elle regroupe les directions fonctionnelles et services les plus impliqués (directions des ressources humaines et des affaires médicales, direction des soins, direction logistique, direction des services techniques, direction des équipements, responsable de la sécurité générale, direction de la qualité et de la gestion des risques, direction informatique, etc.) et les principaux responsables médicaux immédiatement concernés : le président de la Commission médicale locale d'établissement, le chef du service des urgences, le responsable du département d'anesthésie-réanimation notamment. Ces derniers n'ont pas été physiquement présents en cellule de crise, du fait de leur mobilisation pour la prise en charge directe des victimes. Ce sont les membres de la cellule de crise qui ont été amenés à se déplacer sur les lieux de soins 


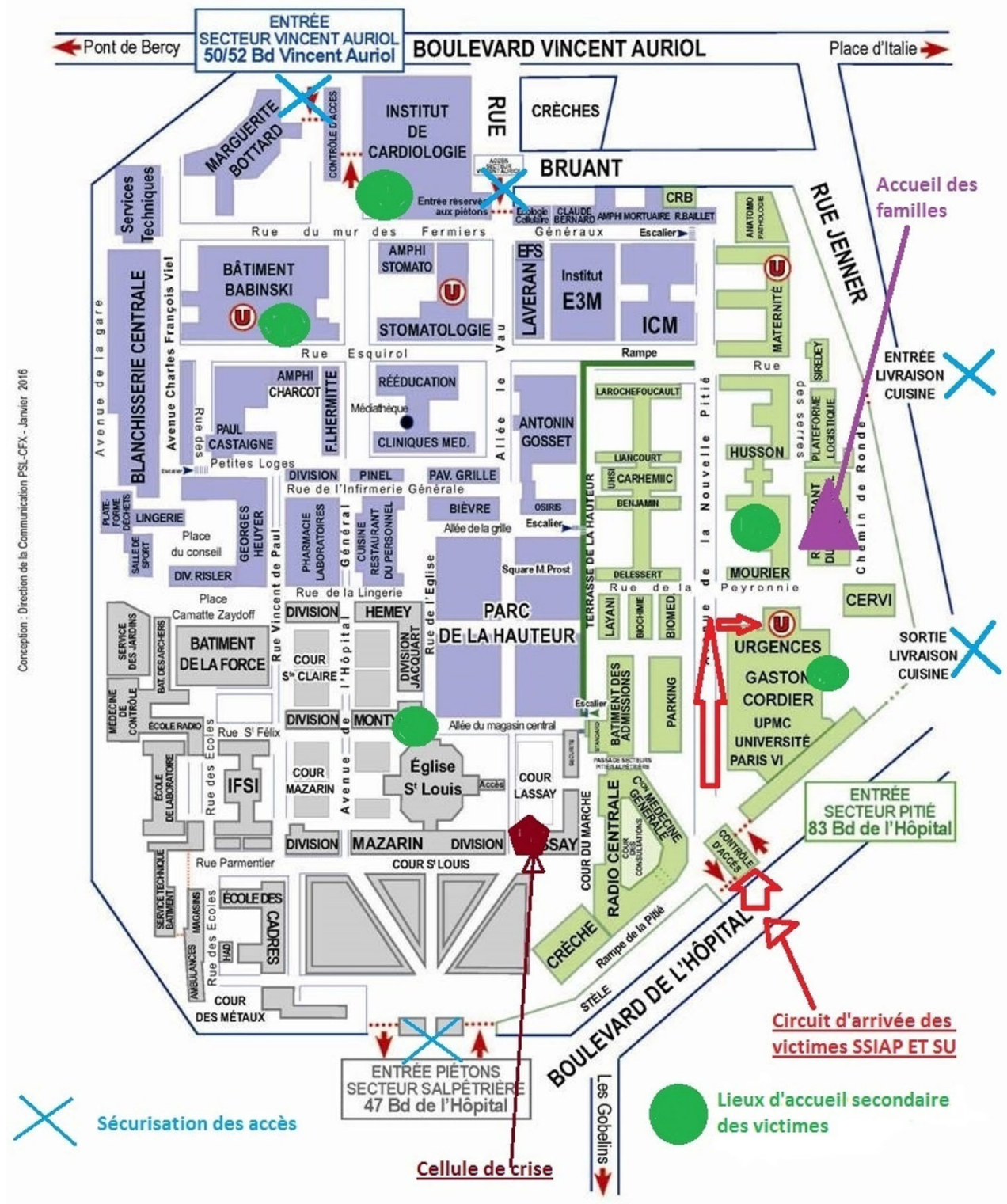

Fig. 1 Mise en œuvre du Plan Blanc sur le site de la Pitié-Salpêtrière

afin de ne pas mobiliser inutilement du temps médical en de telles circonstances.

Le mode d'activation de la cellule de crise, décrit dans le Plan Blanc du site, est basé sur un message SMS automatique envoyé, dès l'alerte, par un membre de l'équipe de direction à la cellule ainsi qu'aux chefs de pôles et aux cadres paramédicaux, puis sur une communication par mail des principales directives. Du fait de la mobilisation spontanée, cette organisation n'a été que partiellement nécessaire et par conséquent mise en œuvre. Avant même le déclenchement officiel du Plan Blanc, le directeur du groupe hospitalier avait ainsi réuni autour de lui (Tableau 1) l'ensemble de son équipe et réparti les rôles de chacun, conformément aux fonctions clés décrites dans le Plan Blanc (ressources humaines, logistique, installations techniques, équipements médicaux, sécurisation générale du site et contrôle des accès, communication...).

Conformément au Plan Blanc, la cellule de crise dispose des extractions des fichiers informatiques comportant les coordonnées des personnels médicaux et paramédicaux de l'hôpital, actualisées mensuellement, et accessibles de l'ensemble des postes informatiques qui lui sont strictement dédiés et stockés dans une armoire sécurisée en dehors des périodes d'activation du Plan Blanc. Avant même le recours à ces fichiers, l'équipe en charge de l'accueil des victimes (salle de surveillance post-interventionnelle et d'accueil 
Tableau 1 Personnel présent en cellule de crise.

\begin{tabular}{|c|c|c|}
\hline Personnels présents & Fonctions & Effectifs \\
\hline $\begin{array}{l}\text { Directeur du groupe hospitalier } \\
\text { Directrice adjointe } \\
\text { Directrice de la qualité, gestion des risques et relations } \\
\text { avec les usagers }\end{array}$ & $\begin{array}{l}\text { Coordination de la cellule de crise et de la mise en œuvre } \\
\text { du Plan Blanc }\end{array}$ & 3 \\
\hline Directrice des services économiques et logistiques & $\begin{array}{l}\text { Organisation des fonctions logistiques : } \\
\text { approvisionnements (services cliniques, zone d'accueil } \\
\text { des personnels), gestion de l'accueil physique des familles }\end{array}$ & 1 \\
\hline $\begin{array}{l}\text { Directrice et Directeur adjoint des affaires médicales } \\
\text { Directeur des ressources humaines } \\
\text { Coordinatrice générale des soins et Directeurs des soins }\end{array}$ & $\begin{array}{l}\text { Mobilisation des ressources humaines médicales et non } \\
\text { médicales }\end{array}$ & 6 \\
\hline $\begin{array}{l}\text { Directeur des investissements } \\
\text { Directeur des équipements }\end{array}$ & $\begin{array}{l}\text { Soutien technique à la cellule de crise (équipements, } \\
\text { interventions techniques) }\end{array}$ & 2 \\
\hline Responsable de la sécurité générale & Sécurisation du site & 1 \\
\hline Administrateurs et directeurs de garde, ingénieurs & $\begin{array}{l}\text { Soutien à la cellule de crise : réponse téléphonique } \\
\text { aux familles, accompagnement des familles sur les lieux } \\
\text { d'hospitalisation des victimes, gestion des flux } \\
\text { logistiques... }\end{array}$ & 8 \\
\hline Directrice adjointe de la communication & Communication & 1 \\
\hline Responsable du standard et des accueils & $\begin{array}{l}\text { Organisation de l'accueil téléphonique et physique } \\
\text { des familles à la recherche de proches (mobilisation le } 14 \\
\text { au matin) }\end{array}$ & 1 \\
\hline Pharmaciens & $\begin{array}{l}\text { Soutien logistique : médicaments, dispositifs médicaux, } \\
\text { stérilisation }\end{array}$ & 3 \\
\hline
\end{tabular}

des polytraumatisés [SSPIAP] et service des urgences [SU]) et la cellule de crise ont procédé au rappel des personnes ressources-clés, qu'il s'agisse de chirurgiens, de cadres de blocs opératoires notamment. Ces derniers ont ensuite sollicité directement leurs équipes et la cellule de crise est intervenue en appui afin d'étendre les rappels à certaines fonctions prioritaires : infirmiers de bloc opératoire diplômé d'État (IBODE) et aides-soignants (AS) de bloc opératoire, ambulanciers, agents de stérilisation notamment.

Compte tenu de l'évolutivité de la situation (l'assaut n'avait pas encore été donné au Bataclan), les rappels de personnel ont été massifs. La décision d'ouverture d'une zone d'hébergement destinée aux professionnels a parallèlement été prise, conformément aux dispositions prévues dans le Plan Blanc (voir chapitre « Activation des fonctions supports »).

\section{Discussion et analyse des points d'amélioration}

On notera que le jour et l'heure de survenue de l'événement ont été particulièrement favorables à une mobilisation rapide et massive des professionnels, grâce notamment au relais de l'information liée aux attentats dans les médias et sur les réseaux sociaux. Bien que d'un impact limité, du fait précisé- ment de ces circonstances favorables, la diffusion d'une alerte SMS automatique apparaît pertinente. La liste de diffusion de l'alerte a depuis été encore élargie (aux chirurgiens, pharmaciens et cadres des secteurs les plus concernés). Le bilan de la mobilisation des professionnels apparaît globalement positif : le retour spontané, doublé d'un rappel ciblé, a permis de répondre à l'intégralité des besoins en termes de prise en charge des victimes. On notera même qu'une fois la situation stabilisée, il y a eu probablement plus de professionnels présents que nécessaire.

Néanmoins, il est apparu plusieurs points de vigilance pour l'avenir :

Les listes de professionnels issues des logiciels de gestion des ressources humaines ont le mérite d'être à jour en termes de mouvements mais ne comportent pas d'informations précises sur les compétences spécifiques acquises qui peuvent s'avérer nécessaires en cas de rappel massif (exemples : aptitude à manipuler des engins de manutention, à conduire des camions ou des ambulances, manipulateur de radiologie formé à l'utilisation d'un scanner, expérience récente en réanimation ou au bloc opératoire...). Cette limite doit nous conduire à identifier plus précisément ces fonctions potentiellement utiles et à établir des listes de personnels compétents en lien avec les cadres paramédicaux des pôles 
cliniques et médicotechniques et les directions fonctionnelles concernées.

Les contacts avec les familles et les proches des victimes (standard téléphonique, accueil) ont présenté une charge émotionnelle extrêmement forte, d'autant qu'il était souvent difficile de délivrer une information fiable (voir le chapitre "Identification des victimes, information des familles »). Ces tâches ont souvent été confiées à des personnels administratifs et techniques rapidement disponibles (personnels logés notamment). Rétrospectivement, ce choix n'était probablement pas le plus pertinent, d'autant que des personnels médicaux ou paramédicaux non mobilisés pour l'événement (services de médecine par exemple) avaient proposé leur aide. Il apparaît donc nécessaire d'établir plus précisément des profils de personnels en fonction des compétences recherchées et de renforcer particulièrement l'accueil afin d'offrir le plus de disponibilité et de compétence possible aux familles et aux proches. Cette mobilisation doit par ailleurs se concevoir dans la durée, durant probablement les $48 \mathrm{~h}$ qui suivent l'événement.

La mobilisation de nos concitoyens, professionnels de santé ou non, actifs ou retraités, a été importante. Néanmoins, cette manifestation de solidarité nécessite un cadrage précis en amont afin d'affecter les personnes à des tâches adaptées à leur profil.

Le rappel et/ou le retour spontané des professionnels n'ont pas été suffisamment intégrés en termes d'impacts sur la gestion des plannings dans les jours qui ont suivi l'événement. Ce point suggère de renforcer la gestion prévisionnelle des ressources humaines pendant la crise en croisant de manière systématique les listes de personnels rappelés et les plannings afin d'éviter l'épuisement et donc la non-disponibilité de ressources, en cas d'activation prolongée du Plan Blanc. Il faudra donc définir à l'avenir une durée de mobilisation maximale des personnels (évaluée à $6 \mathrm{~h}$ ) au-delà de laquelle ils devront partir se reposer et être remplacés car, fortement investis dans l'action dans de telles circonstances, ils n'en ont pas l'initiative spontanément. Il faut donc penser à planifier les ressources humaines et les compétences dans la durée en évitant de mobiliser trop de forces au début de la crise et en assurant la continuité au fil des jours, si besoin. Ceci passe par une information en amont, auprès des personnels hospitaliers, pour qu'ils le comprennent et l'acceptent. Il est par ailleurs nécessaire d'assurer une traçabilité rigoureuse des personnes mobilisées, particulièrement dans l'hypothèse où des restrictions de circulation drastiques viendraient à être mises en œuvre.

Enfin, l'éventualité d'une coupure du réseau de téléphonie mobile en cas d'attaque terroriste de très grande ampleur a été prise en compte en s'assurant de la présence des coordonnées de téléphone fixe dans les listings de personnel.

\section{Activation des fonctions supports}

\section{Organisation et mise en ouvre}

Du fait de la présence des acteurs clés du dispositif décrit précédemment, les fonctions supports ont été mises en œuvre extrêmement rapidement, conformément aux principes définis dans le Plan Blanc.

En ce qui concerne la sécurisation du site, 2 vigiles sont arrivés en renfort des 11 déjà présents, portant leur nombre total à 13 personnes dès $23 \mathrm{~h}$ le 13 novembre 2015 . Les actions de sécurisation du site ont permis, d'une part de renforcer les contrôles d'accès et faciliter l'arrivée des véhicules de secours, et d'autre part, de sécuriser le bâtiment où ont été accueillies les victimes. L'accès principal du bâtiment a ainsi été fermé et 5 vigiles ont été positionnés au sein du service des urgences, de la salle de surveillance post-interventionnelle et d'accueil des polytraumatisés ainsi qu'à l'entrée de la zone lits portes/réanimation.

Un lieu d'accueil spécifique des familles et des proches a été ouvert dans un des restaurants du personnel. Une signalétique dès l'entrée de l'hôpital a été apposée pour faciliter l'orientation vers cette structure. Des professionnels mobilisés (principalement des personnels administratifs et techniques) ont été affectés à cette zone, dans l'attente de la cellule d'urgence médicopsychologique. La direction a assuré l'accueil dans les meilleures conditions possibles en proposant nourriture et boisson aux personnes présentes.

Une zone d'accueil, dans une unité d'hospitalisation de semaine inoccupée, a été offerte aux professionnels afin qu'ils puissent s'y reposer en cas de besoin après leur intervention.

L'ampleur de l'accueil des victimes a nécessité de mettre en œuvre très rapidement un soutien logistique à la prise en charge médicale, axé principalement sur le réapprovisionnement en continu des médicaments, des dispositifs médicaux (DM) et sur le renforcement de la fonction stérilisation (du fait de l'intense activité chirurgicale). Immédiatement après l'alerte, la cellule de crise a mobilisé 3 pharmaciens, dont le chef de service, en renfort de l'interne de garde de pharmacie. La cellule de crise a centralisé durant la nuit toutes les demandes au fil de l'eau et assuré les livraisons, en lien avec les pharmaciens présents et les personnels techniques mobilisés. Le lendemain matin (à J1), les pharmaciens ont alerté leur centrale d'achat (AGEPS) afin de permettre un réapprovisionnement dès le lundi (à J3) des produits de santé les plus prescrits pendant la crise (médicaments et dispositifs médicaux). Un réapprovisionnement en urgence des dispositifs médicaux implantables a toutefois dû être réalisé durant le week-end. 


\section{Discussion et analyse des points d'amélioration}

Le bilan est très satisfaisant et la majorité des dispositions du Plan Blanc a été mise en œuvre conformément aux principes définis. En premier lieu, la sécurisation des lieux d'accueil des victimes et l'ouverture d'une zone dédiée aux familles, à distance de la prise en charge, se sont avérées essentielles afin de permettre aux professionnels de travailler dans la sérénité.

S'agissant de la logistique, on notera, une nouvelle fois, que la qualité du résultat est le fruit d'une mobilisation très importante des professionnels et notamment des principaux « experts métiers » (directeur des services économiques et logistiques et pharmaciens tout particulièrement) dont la connaissance des procédures et du site (très complexe et étendu) a été déterminante. Il est par conséquent nécessaire d'anticiper des conditions moins favorables (absences durant les congés scolaires par exemple) en simplifiant autant que possible la gestion des stocks mobilisables (identification, regroupement). S'agissant enfin tout particulièrement des produits de santé, il apparait nécessaire de porter la réflexion sur quatre axes :

- définir précisément la liste de dispositifs médicaux stérilisables, à haut risque de rupture (rotation insuffisante du processus de stérilisation) en cas d'afflux massif de blessés et rechercher leur équivalent en usage unique ;

- réévaluer les stocks minimaux de certains DMS ;

- renforcer le contact avec les principaux fournisseurs de DMS hors stock APHP pour envisager une livraison rapide en situation de crise ;

- réévaluer également le stock de médicaments « incontournables » à la lumière des besoins identifiés durant la crise

\section{Identification des victimes, information des familles}

\section{Organisation et mise en ouvre}

Dès l'information sur les attentats, les familles se sont adressées aux différents hôpitaux parisiens pour tenter d'obtenir des nouvelles de leurs proches. Cette situation a été sans conteste la plus complexe à appréhender. On ne reviendra pas sur les principes d'identification préhospitalière par le système « SINUS », destiné à assurer, en temps réel et tout au long de la chaîne de soins, le suivi des victimes. L'AP-HP dispose, en complément, d'un outil interne, « VICTIMES », destiné à consolider les données de tous ses hôpitaux (identité des victimes, lieu d'hospitalisation). Dans la nuit du 13 novembre, de nombreuses victimes accueillies (près de la moitié) ne possédaient pas d'identité et n'étaient pas en capacité de la révéler compte tenu de la gravité de leur état.
La situation s'est progressivement améliorée et, le 15 novembre au soir, l'établissement a fiabilisé toutes les identités des victimes hospitalisées. L'identification des victimes a été réalisée " avec les moyens du bord » grâce à l'ingéniosité des professionnels (interrogation des portables des victimes par exemple) et l'interrogation des familles et des proches (recherche de signes distinctifs, bijoux...). De nombreuses photos des personnes recherchées ont ainsi été échangées entre la cellule de crise, les médecins réanimateurs et les professionnels chargés de l'accueil physique des familles. L'adresse mail de la cellule de crise a été communiquée aux familles par téléphone afin de recueillir et diffuser des photos des victimes potentielles.

En parallèle de l'accueil physique des familles dans un lieu dédié, la cellule de crise a organisé l'accueil téléphonique, en complément des numéros d'appels communiqués par les pouvoirs publics. Dès confirmation de l'identité des victimes, les familles ont été informées, soit directement sur place par un des professionnels présents sur le lieu d'accueil dédié, soit par téléphone quand elles avaient déjà été enregistrées. Plusieurs membres de la cellule de crise ont été chargés, après accord des équipes médicales en charge des patients, d'accompagner les familles au chevet de leur proche (service des urgences et lieux d'accueil secondaire pour les victimes opérées).

\section{Discussion et analyse des points d'amélioration}

La problématique d'identification des victimes a été à l'origine de très nombreuses difficultés :

En matière d'identitovigilance : les corrections d'identité des victimes au fil des heures ont généré de nombreux dysfonctionnements dans le système d'information. Plusieurs doublons de dossiers ont ainsi été créés causant des difficultés majeures d'accès aux informations médicales (examens d'imagerie médicale, biologie). Ces dysfonctionnements ont justifié, dans la nouvelle version du Plan, une révision complète des principes d'identification des victimes à l'arrivée avec création d'un $\mathrm{n}^{\circ}$ unique sur le serveur d'identité en lien avec le $n^{\circ}$ «SINUS».

En matière d'identification des victimes : les recherches d'identité ont été conduites par les professionnels du site, sans aucun accompagnement des autorités judiciaires, malgré les demandes répétées de la direction de l'établissement. Les recherches ont ainsi été laborieuses, parfois insuffisamment rigoureuses, faute d'expertise dans ce domaine. Chacun avait par ailleurs à cœur de donner rapidement un nom aux victimes pour permettre l'information des familles. On notera que pour l'une des trois victimes décédées, l'identification formelle n'a été confirmée que trois jours après le décès. Si le recours aux services spécialisés de police judiciaire devait demeurer difficile à obtenir rapidement, il conviendrait de trouver à l'avenir (ce point est en discussion 
dans le cadre des retours d'expérience à l'échelon régional) des solutions locales pour améliorer l'identification des victimes, notamment par la formalisation d'un guide d'interrogation des familles et des proches, à partir de questions adaptées.

Conséquence directe des difficultés d'identification, les relations avec les familles sont à l'origine d'un très fort sentiment d'impuissance des professionnels et plus particulièrement de la cellule de crise qui a géré la réponse téléphonique et l'accueil physique de centaines de proches tout au long du week-end. Localement, les points suivants doivent être améliorés :

Soutien psychologique : la cellule d'urgence médicopsychologique s'est mise en place assez tardivement. Compte tenu des ressources disponibles sur le site (deux services de psychiatrie, de nombreux psychologues), il semble nécessaire de formaliser dans le Plan Blanc une organisation locale, en complément de la mobilisation des cellules d'urgence médicopsychologique (CUMP) qui ont été très sollicitées.

Information rapide du dispositif d'accueil mis en œuvre : une page sur le site internet du groupe hospitalier permettant de diffuser toutes les informations utiles aux familles en cas d'attentats (lieux d'accueil, numéros d'appels, adresse mail pour l'envoi de photos) a été créée de manière à être activée en cas de besoin.

Information des familles : ce point est le plus sensible et dépasse le site de la Pitié-Salpêtrière. Largement évoqué lors des retours d'expérience avec d'autres hôpitaux parisiens, le sujet est complexe. Les pouvoirs publics ont opté pour la centralisation de l'information mais celle-ci a été en partie défaillante. En effet, d'une part les ressources mobilisées pour l'accueil téléphonique se sont avérées insuffisantes et d'autre part, l'information a tardé à remonter des établissements. Chaque établissement a ainsi été contraint d'organiser sa propre réponse aux demandes, pressantes, des familles, à partir de ses seules données d'information.

Information sur les personnes décédées : en ce domaine, l'établissement s'est trouvé confronté à une situation très difficilement gérable. Les corps des victimes décédées à l'hôpital n'ont pas été transférés rapidement à l'Institut médicolégal pour permettre leur identification (et sans qu'un délai puisse être donné) et en parallèle, pendant trois jours, le parquet s'est opposé à leur présentation aux familles au sein de la chambre mortuaire. Les familles des victimes concernées ont été très affectées par cette situation, jugée incompréhensible y compris par les personnels hospitaliers.

La réponse à ces dysfonctionnements dépasse largement le cadre de la réflexion locale au sein de l'établissement [5]. Elle nécessite en effet qu'un accord soit trouvé entre l'AP-HP et les pouvoirs publics compétents (justice, police, santé) concernant la conduite à tenir à l'avenir, notamment en matière de centralisation de l'information, d'aide à l'identification des victimes, dans le respect de l'instruction diligentée par la justice.

\section{Période post-crise}

La période post-crise a été marquée par trois actions principales :

- le retour d'expérience permettant à tous les professionnels d'exprimer leur ressenti sur la gestion de la crise et d'en tirer les principaux axes d'amélioration : ces retours ont été menés à plusieurs niveaux. Une réunion très large associant l'ensemble des professionnels médicaux et paramédicaux ainsi que des membres de la direction impliqués dans la gestion de la crise a été organisée dix jours après les événements. Cette réunion a été d'une très grande richesse tant sur le plan du retour d'expérience et de l'identification partagée des axes d'amélioration que sur le plan psychologique en termes de valorisation des équipes et d'expression des professionnels sur des événements particulièrement traumatisants. Des retours d'expérience plus ciblés ont également été organisés au sein des différentes structures impliquées (SSPIAP, SU, réanimations) et de la direction. Tous les axes d'amélioration identifiés ont été, ou seront, très rapidement intégrés dans les nouvelles versions des plans concernés (Plan Blanc et Plan NoVi-H) ou directement dans les procédures de services ;

- il est apparu très rapidement la nécessité d'apporter un soutien psychologique aux équipes impliquées à tous les niveaux. De nombreux groupes de parole ont été organisés dans les services concernés et une cellule de soutien a été mise en place au sein de l'hôpital par le service de psychiatrie en concertation avec la direction des ressources humaines et le service de santé au travail. Une note d'information comportant une grille d'évaluation du stress post-traumatique a été proposée afin de permettre aux professionnels d'identifier et de quantifier le cas échéant leur niveau de souffrance psychologique afin de recourir si besoin à un psychiatre ou un psychologue ;

- la coopération avec les autorités policières, dans le cadre de l'enquête. Dans ce domaine, on insistera sur deux points : d'une part la nécessité d'un rappel préalable aux services de police (avec qui la collaboration a été bonne) des règles à respecter (communication des réquisitions en bonne et due forme et demande des autorisations médicales préalables aux auditions des victimes hospitalisées notamment) et d'autre part, la centralisation par un membre de l'équipe de direction des contacts avec les services de police afin de répondre de manière cohérente aux multiples sollicitations, concernant notamment la liste des victimes mise à jour, l'organisation des auditions in situ, le regroupement des pièces à convictions, etc. 


\section{Conclusion}

Ces dernières années, la direction s'est particulièrement impliquée dans la mise à jour et l'ajustement de ses plans de crise. Déjà très mobilisé par la problématique d'origine infectieuse (la Pitié-Salpêtrière est le site référent pour l'accueil des patients potentiellement infectés par les virus Ebola et Mers-Corona), l'établissement avait réfléchi, en parallèle, et compte tenu du risque terroriste majeur dramatiquement confirmé par les événements précédents de janvier 2015 à Paris, à l'amélioration de son Plan Blanc, au travers d'une simplification des procédures et d'exercices de simulation de déclenchement. Jamais dans son histoire l'établissement n'a dû faire face à une telle situation et la mobilisation de tous les personnels (médecins anesthésistesréanimateurs et réanimateurs médicaux, chirurgiens, radiologues, cadres de santé, infirmières, aides-soignants, personnels administratifs, techniques et logistiques) a été remarquable et constitue une véritable fierté collective, au service de nos concitoyens. Les attentats du 13 novembre 2015 nous ont tous encore plus convaincus de la nécessité d'avoir des plans de crise bien établis, clairs, actualisés régulièrement, connus des personnels hospitaliers, et de s'y entraîner régulièrement même si cela peut perturber le fonctionnement courant de l'hôpital. Fort de cette nouvelle expérience tragique, il s'agit désormais de faire encore évoluer le Plan Blanc à la lumière des points faibles identifiés. Il convient également de s'inscrire dans une logique d'anticipation en approfondissant d'autres scenarii de crise, à savoir un possible attentat chimique ou bien même une attaque terroriste au sein même de l'hôpital.

Liens d'intérêts : Les auteurs ont déclaré ne pas avoir de lien d'intérêt.

\section{Références}

1. Hirsch M, Carli P, Nizard R, et al (2016) The medical response to multisite terrorist attacks in Paris. Lancet 369:386:2535-8

2. Le Premier ministre (2013) Décret $n^{\circ} 2013-15$ du 7 janvier 2013 relatif à la préparation et aux réponses aux situations sanitaires exceptionnelles. http://www.legifrance.gouv.fr/affichTexte.do?cidTexte=JORFTEXT000026915951 (Dernier accès le 30 décembre 2015)

3. Direction de l'hospitalisation et de l'organisation des soins (DHOS) (2002) Circulaire DHOS/HFD n²002/284 du 3 mai 2002 relative à l'organisation du système hospitalier en cas d'afflux de victimes. www.sante.gouv.fr/IMG/pdf/circulaire_du_2 mai_2003-2.pdf (Dernier accès le 30 décembre 2015)

4. Ministère de la Santé et des Solidarités (2006) Guide d'aide à l'élaboration des Plans Blancs élargis et des Plans Blancs des établissements. Édition 2006, annexe à la circulaire $\mathrm{N}^{\circ} \mathrm{DHOS} / \mathrm{CGR} /$ 2006/401du 14 septembre 2006 http://www.sante.gouv.fr/IMG/pdf/ plan_blanc_2006.pdf (Dernier accès le 30 décembre 2015). http://www.sante.gouv.fr/IMG/pdf/cir_38043.pdf (Dernier accès le 30 décembre 2015)

5. Le Premier ministre (2015) Instruction interministérielle relative à la prise en charge des victimes d'actes de terrorisme 12 novembre 2015. http://circulaire.legifrance.gouv.fr/pdf/2015/11/cir_ 40245 . pdf (Dernier accès le 30 décembre 2015) 